\title{
Etude Pratique Sur Le Principe De La Presomption D'innocence Face Aux Delits De Presse En Droit Positif Congolais
}

\author{
Menga Rene Yaosuwa* and Imani Gaya
}

The University of BUNIA in Acronym UNIBU

DOI: $10.36348 / \mathrm{sb} .2020 . v 06 i 01.006$

| Received: 21.01.2020 | Accepted: 28.01.2020 | Published: 30.01 .2020

*Corresponding author: Menga Rene Yaosuwa

\section{Abstract}

"Il n'est pas possible de réduire l'activité tumultueuse des humains à un ordre géométrique exempt d'irrégularité et de confusion. De même que les lois simples et constantes de la nature ne peuvent éviter les perturbations qui surviennent dans le cours des planètes, les lois humaines sont incapables d'empêcher le trouble et le désordre résultant des forces d'attraction innombrables et opposées du plaisir et de la douleur. C'est pourtant la chimère que poursuivent les hommes aux facultés limitées quand ils ont en main le pouvoir. Lorsqu'on défend une foule d'actes indifférents, on ne prévient pas des délits qui ne sauraient en résulter, mais on en crée de nouveaux en déplaçant arbitrairement, entre le vice et la vertu, des limites que l'on proclame cependant éternelles et immuables. (...) Si l'on veut prévenir les délits, il faut faire en sorte que les lois soient claires et simples, et que tous les membres de la nation unissent leurs forces pour les défendre, sans qu'aucun ne puisse travailler à les détruire."

[Cesare Beccaria, Des délits et des peines, Librairie Droz, Genève, 1965, § XLI. Moyens de prévenir les délits; Flammarion, Paris, 1991, p. 169].

Copyright @ 2020: This is an open-access article distributed under the terms of the Creative Commons Attribution license which permits unrestricted use, distribution, and reproduction in any medium for non-commercial use (NonCommercial, or CC-BY-NC) provided the original author and source are credited.

\section{Problematique}

La société étant l'apanage de la nature humaine; les hommes sont invités à une vie sociétale pour atteindre l'idéal de la communion. En ce sens, pour une vie harmonieuse au sein des membres de la société, ces derniers doivent avoir des égards aux normes et règles régissant la communauté pour la préservation de l'ordre public.

Notre constitution du 18 févier 2006 telle que révisée à ce jour le prévoit en consacrant une grande importance aux libertés individuelles et en leur réservant tout un chapitre allant de l'article 11 à l'article 33.

La problématique qui se pose est celle de savoir comment et à quel moment l'agent qui occasionne le trouble à l'ordre peut-il répondre de ses actes? Alors que les méandres du droit procédural pénal posent le principe selon lequel «l'auteur de l'infraction ne subit la peine que lorsqu' il a été condamné après avoir été juge par une juridiction instituée à cet effet $\gg[1]$.
L'article 16 de la constitution du 18février 2006 telle que révisée et complétée à ce jour parle «du caractère sacré de la personne humaine ; l'obligation de la respecter; et de la protéger» et poursuit encore que «toute personne a droit à l'information».

Au terme de cette affirmation le principe de la présomption d'innocence trouve son fondement juridique aux articles 17 in fine et 24 de la constitution qui dispose que «Toute personne accusée d'une infraction est présumée innocente jusqu'à ce que sa culpabilité soit établie par un jugement définitif ». Ainsi la préoccupation qui nous vient à l'esprit est celle de savoir comment doit être traité le journaliste accusé de délit de presse? Quelle attitude nos journalistes doivent ils avoirs dans l'exercice de leurs fonctions pour qu'ils ne tombent pas dans la délinquance liée aux medias?

\section{HYPOTHESE}

Avant de parler de la responsabilité de «medias », nous allons comprendre ce que veut dire le délit de presse. Le mot délit vient du latin «delitum» qui signifie péché. Lorsqu'on prend le préfixe de et on l'associe au participe passé delinquere qui signifie 
laissé, délaissé ou renoncé, on a le verbe delinquere qui vent dire «manqué à son devoir, péché ou offenser». C'est de ce mot latin delinquere que vient le mot délinquance ou délinquant.

Le délit est civil mais dans le langage courant, le délit est souvent synonyme d'infraction en droit français par exemple est considéré comme délit, toute infraction que les lois punissent de peine correctionnelle.

Mais en droit congolais, les juristes soutiennent que le concept délit ne relève que de la matière civile précisément a l'art 258 Code Civil Congolais Livre III qui dispose que «tout fait quelconque de l'homme qui cause à autrui un dommage, oblige celui par la faute duquel il est arrivé à les réparer ».

Ici, la loi congolaise ne prévoit ni une liste, ni un contenu précis, il est tel du seul moment qu'il cause préjudice à autrui. Mais En République Démocratique du Congo lors qu'on parle de délit de presse on doit vite recourir à l'art 74 de la loi 96-002 du 22 juin 1996 fixant les modalités de l'exercice de la liberté de presse, pour en tirer la définition. L'article cité définit le délit de presse comme « toute infraction écrite ou audiovisuel ».

AKELE ADAU PIERRE, décèle dans le chef du législateur Congolais, une vision très large du délit de presse. Il affirme que « celui-ci incrimine en quelque sorte l'abus de liberté de la presse c'est-à-dire l'usage de média à de fins de commettre quelques infractions que ce soit ».

Il est le définit comme un délit d'opinion par les moyen de la presse. Pour lui, chaque fois que la presse sert de moyen d'expression à une infraction, cette infraction devient un délit de presse.

Ce qui va nous intéresser plus est l'infraction de la diffamation qui repose sur l'art 74 du code pénal ordinaire. Il y a diffamation lorsque des parole ou des écrits, ont causé du tort à la bonne réputation d'une personne » (Art 74 du code pénal Congolais). Cela est aussi prévu à l'art 77 de la loi du 22 juin 1996 s'agissant notamment de l'offense au chef de l'Etat.

Les hommes de medias sont la plus part de cas passibles de l'infraction de diffamation. Qu'en est-il de cette prévention.

En droit congolais, les imputations dommageables ou la diffamation sont définis comme : « le fait d'imputer à quelqu'un des faits précis, vrais ou faux, à même de porter atteinte à l'honorabilité de ce dernier » Cette catégorie d'infraction est réprimée par les articles 74, 75 et 77 du code pénal ordinaire.
La diffamation suppose « l'imputation d'un fait précis de nature à porter atteinte l'honneur ou à la considération d'une personne ou à l'exposer au mépris ».

Les imputations dommageables ou diffamation ont des éléments constitutifs. Deux éléments essentiels peuvent être retenus, il s'agit de la publicité et de la catégorie des personnes protégées.

Le code pénal congolais ne donne pas la définition de la publicité, il faut comprendre par le terme publiquement en l'employant dans le sens usuels "en public", c'est-à-dire en présence de plusieurs personnes.

La publicité est définie, à en croire LIKULIA BOLONGO « d'après les circonstances et les lieux. Ainsi, la publicité peut résulter soit de propos proférés, soit d'écrits ou images distribués, vendus ou exposés dans les lieux ou réunions publics ».

Par ailleurs, « par lieux publics, on entend outre les lieux publics par nature, c'est-à-dire affectés à l'usage de tout et accessible à chacun à tout moment (voie publique), les lieux publics par destination (bureau, salle d'audience, salle de cours et tribunaux, bars) ouvert au public à certain moment déterminé et aussi les lieux publics par accident, privé en principe mais devenant occasionnellement public par le fait de la présence d'un certain nombre des personnes». Les personnes protégées sont les particuliers.

Quant aux faux bruits, cette infraction est mentionnée à l'art 199 du code pénal Congolais est souvent associée à celle d'imputation dommageable. L'offense au chef de l'Etat ou outrage à l'autorité légalement établis est aussi souvent évoquée lorsque le journaliste est poursuivi pour avoir porté atteinte à la sureté de l'Etat ou pour avoir divulguer des secrets de défense nationale.

Il sied de noter que la liberté de presse n'est pas une valeur absolue. Si le droit garanti a tous citoyens la libre circulation des moyens de communication, il en fixe aussi les limites. Ainsi pour des raisons de précision, selon FRANCIS BALL [2], «l'existence de certaines limites ne sont pas contraire entant que telle, à l'affirmation juridique de la liberté d'expression, la liberté ne pas une licence. Il n'est guère de système juridique qui ne permettent, expressément, de limiter l'exercice des libertés affirmées. Mais ces limitations autres leurs caractères explicites est déterminé, ne peuvent à leurs tour dépassé certaines limites, faute de quoi l'espace de la liberté se réduit comme une peau de chagrin. 
Ces limites ou ces bornes méritent d'être considéré sous un double aspect celui de leur motif ou de leur justification. Ensuite, celui de modalité ces limites ou des procédures pour en garantir le respect.

Dans le même fil d'idée, Luc ADOLPHE TIAO [3], se pose la question sur la responsabilité des médias «faut-il pour autant dédouaner les journalistes et leurs médias lorsqu'ils commettent des erreurs ou des fautes plus ou moins graves? Assurément non. Quand bien même on est un défenseur intrépide de la liberté de la presse.

On doit reconnaitre la part de la responsabilité des journalistes dans les sanctions qui sont prises à leur encontre »où nous faisons référence à la loi congolaise déterminant le mot délit, les juristes relèvent que le concept délit ne relève qu'en matière civile d'où la référence est à l'article 258 CCC L III du décret du 30 Juillet 1888 portant code des contrats, des obligations conventionnelles et en s'appuyant sur les codes civiles congolais, les médias sont des civilement responsables pour les faits qu'ils auraient commis comme entreprise de diffusion d'information ou comme personne physique lorsqu' il s'agit du journaliste seul ( l'articles 258, 259 CCC L III». La loi est claire en cette matière lorsqu'elle souligne que chacun est responsable de dommage qu'il a causé non seulement par ces faits mais encore par sa négligence ou son imprudence.

\section{CHOIX ET INTERET DE L'ETUDE}

De nos jours un pays ne peut pas être considéré comme « civilisé »et jouir de la considération comme étant un Etat de droit c'est-à-dire un Etat qui garantit et respecte les droits humains s'il ne dispose pas des outils d'information et de communication adéquats.

Notre étude revêt deux intérêts capitaux notamment l'intérêt théorique et pratique. D'une part l'examen des problèmes soulevés par le principe de la présomption d'innocence par rapport à la personne accusée pour une infraction liée aux médias. D'autre part interpellé la conscience des responsables des médias des sanctions qu'ils encourent s'ils ne respectent pas la règlementation en matière de circulation de l'information.

\section{OBJJECTIF DE L'ETUDE}

Cette étude renferme les objectifs suivants:

- Préciser les contours légaux en matière de répression des délits de presse ;

- Définir les attitudes que doivent avoir les journalistes congolais dans l'exercice de leurs fonctions ;

- Instruire les autorités judiciaires congolaises sur la procédure à suivre en matière de poursuite et de répression de délits de presse tout en tenant compte du principe de la présomption d'innocence.

\section{METHODOLOGIE DE L'ETUDE}

L'objet d'un travail scientifique dépend étroitement des méthodes et des techniques utilisées lors de la recherche scientifique.

M. GRAWITZ et PINTO [4], définissent la méthode comme : «l'ensemble d'opérations intellectuelles par lesquelles une discipline cherche à attendre les vérités qu'elle poursuit, les démonte et vérifie».

La méthode est définie encore comme une démarche intellectuelle qui vise d'un coté à établir rigoureusement un objet de science et de l'autre coté à mener le raisonnement portant sur cet objet de la manière la plus rigoureuse que possible[5].

Pour ce faire, nous nous somme servis de la méthode exégétique qui consiste à interpréter les textes des lois. Concrètement elle nous a permis de comprendre des dispositions légales du droit commun régissant le principe de la présomption d'innocence face aux medias en droit positif congolais. Nous nous sommes aussi servis de la méthode sociologique pour comprendre la portée de la délinquance médiatique au sein de la société.

La méthode doit s'appuyer sur certain nombre des procédés qui ne sont autres que les techniques des recherches, définit d'ailleurs comme étant : « un outil, un instrument ou moyen concret utilisé par le chercheur pour récolter ou traiter les informations » [4].

Ainsi à l'élaboration de la présente étude, nous avions utilisé TECHNIQUE documentaire qui nous a permis de confronter les différents ouvrages relatifs à la présente étude.

\section{Subdivision de l'etude}

Pour répondre aux problèmes soulèves par cette étude nous allons la présenter en deux titres. Le premier sera consacré sur une approche analytique et explicative du principe de la présomption d'innocence. Le second portera sur la répression des délits de presse face au principe de la présomption d'innocence. Aux termes de ces titres, une conclusion générale viendra clore notre étude.

\section{Approche analytique et explicative du principe de la presomption d'innocence}

\section{Enonce du principe}

La notion de la présomption d'innocence est une notion qui était ignorée dans l'ancien droit et qui a vu le jour au $18^{\mathrm{e}}$ siècle suite aux idées de certains philosophes de ce siècle dit de lumière. 
La présomption d'innocence a été déclarée ou exprimée avec force dans l'article 9 de la Déclaration universelle des Droits de l'homme et des citoyens du 04 août 1789 et encore dans l'article 11 de la Déclaration Universelle des Droits de l'Homme du 10 décembre 1948, avec une formule célèbre qui d'ailleurs lie étroitement l'innocence présumée et la protection de la liberté individuelle au cours du procès pénal. Ce principe est composé de deux mots: présomption et innocence. Ainsi nous allons parler de la présomption comme étant «une supposition, ou un jugement fondé sur des simples indices de supposition »;

L'innocence veut dire «l'absence de culpabilité, ou la pureté d'une personne qui ignore le mal». En formant ces deux mots, la présomption d'innocence peut être définie comme une supposition de l'absence de culpabilité. En matière pénale il est un principe selon lequel «toute personne poursuivie est innocente des faits infractionnels qui lui sont reprochés, tant qu'elle n'a pas été déclarée coupable par une juridiction compétente».

\section{FONDEMENT}

La présomption d'innocence est un principe qui tire son fondement dans la constitution et dans les autres textes du pays notamment le droit pénal, la procédure pénale, etc. Ainsi, conformément à l'article 17 in fine de la constitution du 18 févier 2006 telle que révisée et complétée à ces jours, et l'article 11 de la déclaration universelle des droits de l'homme «Toute personne accusée d'une infraction est présumée innocente jusqu'à ce que sa culpabilité soit établie par un jugement définitif ». Le droit pénal et la procédure pénale reposent sur le principe de la légalité des délits et des peines.

Ce principe peut aussi s'énoncer de la manière suivante «pas d'infraction, sans peine ».Un tel principe suppose qu'il admet la notion de la peine et d'infraction ainsi que la nécessité d'une répression pour protéger l'ordre public. Le même principe consacre la sauvegarde de la liberté individuelle puisqu'il ajoute «pas d'infraction sans texte ».

Pratiquement, le principe de la légalité qui domine notre droit pénal signifie que toutes les infractions, toute les peines doivent être prévues antérieurement par la loi.

\section{Les beneficiaires du principe de la presomption d'innocence}

La constitution de notre pays du 18 février 2006 telle que révisée à ces jours à son article 17 in fine dispose que «toute personne accusée d'une infraction est présumée innocente jusqu'à ce que sa culpabilité ait été établie par un jugement définitif ». De même le pacte international relatif aux droits civils et politique du 09 décembre 1966 à New York a son article 14.2, ainsi que la convention de sauvegarde des droits de l'homme et libertés fondamentales ne sont pas restés indifférent à cette question pour renforcer ce principe de la présomption d'innocence. Le souhait de tous ces textes est de garantir la protection à la personne accusée et recherchée une infraction avant qu'un jugement définitif ne soit rendu par une juridiction compétente.

Ainsi nous disons que toute personne qui fait encore l'objet d'une simple suspicion et celle qui est jugée et dont le jugement n'est pas encore rendu définitif sont des bénéficiaires du principe de la présomption d'innocence.

\section{Les personnes accusees d'une infraction}

Toute personne accusée d'une infraction peut faire objet d'une détention selon l'économie de l'article $27 \mathrm{du}$ code d'organisation, fonctionnement et compétence de juridictions de l'ordre judiciaire, « s'il existe contre lui des indices sérieux de culpabilité et qu'en outre les faits paraissent constituer une infraction que la loi réprime d'une peine de six mois de servitude pénale au moins». L'alinéa du même article affirme que, la personne contre qui existe des indices sérieux de culpabilité peut être mise en état de détention préventive lorsque le fait parait constituer une infraction que la loi punie d'une peine inférieure à six mois de servitude pénale; mais supérieure à sept jours s'il y a lieu de craindre la fuite aussi au regard des circonstances graves et exceptionnelles, elle est impérieusement réclamée par l'intérêt de la sécurité publique. Ainsi la détention préventive comme son nom l'indique va entrainer l'incarcération de la personne poursuivie. Il s'agit là d'une mesure très grave pour la liberté individuelle et qui parait contraire à la présomption d'innocence. L'intéressé subissant l'équivalent de sa peine, alors qu'il n'a pas encore été condamné ou jugé.

La souveraineté de l'appréciation de la détention est restée à la seule volonté du juge qui décide de la liberté de ces semblables. «La liberté est le principe; la détention est l'exception dit-on. Le juge dans l'exercice des fonctions lui attribuées par la loi, doit prendre en compte les réalités sociologiques et juridiques de son milieu »

\section{Les personnes recherchees}

Nos lois reconnaissent que les organes de poursuite, d'enquête, d'instruction, de mise en accusation et de jugement ont, non seulement les devoirs, mais aussi des très larges pouvoirs de recourir à tout moyen utile pour découvrir, convaincre et permettre de juger justement les auteurs des crimes dans l'intérêt de la protection commune. 
En principe les devoirs et pouvoir de rechercher, de constater des preuves par la police judiciaire sont formellement consacrés. C'est dans ce souci que l'article 2 al.1 du code d'organisation, de fonctionnement et de compétence de juridiction de l'ordre judiciaire dispose ce qui suit : «les officiers de police judiciaire constatent les infractions qu'ils ont pour rôle de rechercher. Ils reçoivent les dénonciations, plaintes, rapports relatifs à ces infractions».

\section{De la repression des delit de presse face au principe de la presomption d'innocence}

Il importe de signaler que la constitution de la République Démocratique du Congo du 18 février 2006 telle que révisée et complétée à ce jour à son article 24 al.1 dispose que «toute personne a droit à l'information » ainsi le même article à son dernier alinéa parle « des médias audiovisuels et écrits d'Etat sont des services publics dont l'accès est garanti de manière équitable à tous les courants politiques et sociaux. Le statut des médias d'Etat est établi par la loi qui garantit l'objectivité, l'impartialité et le pluralisme d'opinion dans le traitement et la diffusion de l'information ». Cette disposition expose la philosophie de l'Etat congolais en matière d'exercice des fonctions des journalistes dans un environnement public.

\section{LES MISSIONS DES MEDIAS \\ Récolte Des Informations}

Le journaliste a comme métier d'informer le public, c'est - à - dire il doit descendre sur terrain pour collecter des informations. Ce qui constitue sa première mission professionnelle. Cela peut se faire personnellement ou par le canal des correspondants.

Comme le dit FRANCIS BALLE [2]« le média est comme une institution sociale (moyen de communication de masse) qui répond aux besoins précis »c'est - à - dire dans chaque territoire du monde, il y a des journalistes (médias) qui font leurs missions de chercher (collecter) les informations pour permettre à l'opinion publique de savoir ce qui se passe dans tel ou tel lieu sur le plan politique, scientifique, culturelle, promotionnelle et même humouristiques.

\section{Traitement des informations}

Toute information doit être traitée. Et cela peut être de plusieurs modèles (le desk, le reportage, la correspondance).

En effet, cette deuxième mission est importante dans le chef du journaliste, elle est souvent déterminée par la nature des médias, l'objectif du papier et la compétence du journaliste.

\section{Diffusion des informations}

Cette étape est étroitement fonction du média, de l'objectif de l'informateur et la nature de média qui joue un rôle important au-delà du fait que l'information demeure une denrée périssable.

Tout journaliste doit diffuser une information qui ne porte pas atteinte aux bonnes mœurs. Ici il y a ce qu'on appelle le quadrillage qui est le contrôle de l'information pour qu'elle soit bien diffusée. Ou celle que l'on peut appeler la désinformation qui consiste à rectifier une information déjà diffusée.

\section{Les droits et devoirs des medias}

Dans toutes les sociétés du monde les hommes sont soumis à certains droits et devoirs pour le bien-être de tous.

\section{Droits des medias: texte de base}

Le journaliste avant d'être un professionnel de la communication médiatique est d'abord une personne humaine.

La constitution consacre certains droits humains qui concernent d'une manière particulière le chevalier de la plume, notamment le droit à la liberté de pensée, droit à la liberté d'expression, droit à l'information, liberté d'information et d'émission par la radio et la télévision, droit à la protection etc.

Les dispositions de la loi $n^{\circ} 96-002$ du 29 juin 1996 abordent quelques droits fondamentaux reconnus aux journalistes aux articles 8-10, section $3 \mathrm{du} 1^{\mathrm{er}}$ chapitre. Nous pouvons citer entre autre: droit à la liberté d'opinion et d'expression, droit à la liberté, à l'indépendance d'esprit, la liberté de choix du titre d'un écrit ou d'une émission.

\section{Les devoirs des medias: textes de base}

La constitution de la RDC contient plusieurs dispositions qui rappellent les devoirs du journaliste. Son article 31 recommande au journaliste de veiller sur «le droit de toute personne au respect de sa vie privée et au secret de la correspondance, de la télécommunication et de toute autre forme de communication ». La constitution insiste également sur le respect de la loi, de l'ordre public, des droits d'autrui et des bonnes mœurs.

Hormis la constitution, les autres devoirs des medias sont fixés par la loi du 30 juin 2004 portant l'organisation, attribution et fonctionnement de la haute autorité des médias.

Dans cette loi organique, la Haute Autorité des Medias, n'institue guère de nouvelles obligations à l'égard du journaliste. Elle revient avec une terminologie un peu moins abstraite sur ses devoirs. D'où après avoir rappelé les obligations constitutionnelles et celles contenues dans la loi de 96, elle formalise certaines obligations conséquentes notamment: respect du pluralisme des courants de 
pensées et d'opinions, préservation de la santé publique et de l'environnement, sauvegarde de l'identité culturelle etc.

En outre, il faudra aussi souligner le fait que la loi portant fonctionnement de la Haute Autorité de Media à outrepasser le secteur de la déontologie et a élargi son champ sur le terrain du droit pénal lorsqu'elle oblige le journaliste de ne pas recourir à «l'incitation à la haine tribale, ethnique ou raciale, à la xénophobie ainsi qu'à toute autre forme de discrimination à travers les médias ».

\section{Les abus des medias violant le principe de la presomption d'innocence}

Selon NYABIRUNGU [6], malgré les droits qu'ont les journalistes d'informer les citoyens et ces derniers d'être informés «il s'agit concrètement d'un conflit entre deux valeurs consacrées aussi bien par les instruments internationaux que par la constitution nationale et dont le défaut de proclamation de la priorité de l'une sur l'autre n'empêche pas qu'à l'occasion des cas d'espèce, l'une de ces valeurs prévale».

Cela veut dire que quel que soit l'importance reconnue au principe de la présomption d'innocence dans l'exercice des fonctions d'un journaliste, il doit avoir ses limites.

Il importe cependant de relever que cette présomption d'innocence parait incompatible avec les dispositions des articles 27 de procédure pénale Congolais et 17 de la constitution du 18 février 2006 telle que révisée et complétée à ce jour qui autorisent la détention préventive lors qu'il existe des indices sérieux de la culpabilité.

Certes, il est vrai que la présomption d'innocence s'efface au fur et à mesure que les indices de la culpabilité pèsent sur la personne. La détention serait une mesure pouvant empêcher l'auteur présumé de l'infraction de se soustraire de la poursuite aussi longtemps qu'il existe des indices sérieux de culpabilité. La poursuite pénale d'un journaliste peut se justifier suivant le prescrit de la constitution qui affirme que «la personne humaine est sacrée, l'Etat a l'obligation de la respecter et la protéger »(Art 16 de la constitution).

Ainsi, le journaliste l'auteur présumé de l'infraction perd le bénéfice de la présomption d'innocence lorsque les faits mis à sa charge portent atteinte à l'honneur ou à la considération de la personne. La critique reste libre et nécessaire dans toutes sociétés se prétendant démocratique comme l'a dit Emanuel DREYER [7], seul élément à prendre en compte est les faits de porter atteinte à l'honneur et à la considération de la personne.
Par ricochet, lorsque l'honneur et la considération d'une personne sont dénaturées par voie médiatique, l'Etat est appelé à intervenir en vertu de la prérogative constitutionnelle qui lui est reconnue par le biais de l'action publique nonobstant le principe de la présomption d'innocence. Les délits de presse portent des stigmates psychologiques, professionnels, sociétaux et familiaux. Les acteurs des medias sont dans l'obligation de moyen et de résultat quant à la vérification du contenu des informations qu'ils doivent diffuser.

$\mathrm{Au}$ nombre des medias traditionnels, il faut reconnaitre à ce jour le développement de nouvelles technologies de l'information et de la télécommunication ainsi que l'Internet qui a contribué à l'émergence d'une nouvelle manière de vivre et de nouvelle mentalité.

Il est évident que l'Internet est parmi les meilleures inventions du 21éme siècle dans la mesure où il offre des facilités d'apprentissage et de communication plus rapide et moins couteux. Il constitue en même temps un espace pour toutes sortes d'activités. Les opportunités inépuisables offertes par l'Internet ne sont toujours pas mises en avant pour un développement harmonieux.

Disons en ce sens que toute invention humaine porteuse de progrès peut être aussi génératrice des comportements illicites. Le coté élogieux de l'internet semble occulter sa face la plus redoutable. Ainsi, un bon nombre des citoyens dits internautes utilisent son côté négatifs par des pratiques en marge de la loi. Soulignons avec force que le journalisme virtuel prend de plus a plus de l'ampleur en République Démocratique du Congo.

Avec le développement de la technologie plusieurs réseaux sociaux ont vu le jour avec comme objectif commun la communication .Il est important de signaler qu'il existe des Réseaux sociaux généralistes ou spécialisés, réseaux sociaux mobiles ou géo localisés, réseaux sociaux professionnels ou anonymes et les réseaux sociaux sur mobile uniquement. Il existe forcément un réseau social adapté à vos besoins : échanges avec vos proches ou avec vos collèges, promotion de votre entreprise, création, publication et partage de contenu.

Les hommes de medias sont la plus part de cas passibles de l'infraction de diffamation sur ces réseaux virtuels de communication. Qu'en est-il de cette prévention et quelle est sa portée sur Internet.

En droit congolais, les imputations dommageables ou la diffamation sont définis comme : « le fait d'imputer à quelqu'un des faits précis, vrais ou faux, à même de porter atteinte à l'honorabilité de ce 
dernier » Cette catégorie d'infraction est réprimée par les articles 74, 75 et 77 du code pénal ordinaire.

En effet, la diffamation suppose « l'imputation d'un fait précis de nature à porter atteinte à l'honneur ou à la considération d'une personne ou à l'exposer au mépris ».Les imputations dommageables ou diffamation ont des éléments constitutifs. Deux éléments essentiels peuvent être retenus, il s'agit de la publicité et de la catégorie des personnes protégées.

Le code pénal congolais ne donne pas la définition de la publicité, il faut comprendre par le terme publiquement en l'employant dans le sens usuels de "en public", c'est-à-dire en présence de plusieurs personnes.

En effet, la publicité est définie, à en croire LIKULIA BOLONGO «d'après les circonstances et les lieux. Ainsi, la publicité peut résulter soit de propos proférés, soit d'écrits ou images distribués, vendus ou exposés dans les lieux ou réunions publics ».

Par ailleurs, «par lieux publics, on entend outre les lieux publics par nature, c'est-à-dire affectés à l'usage de tout et accessible à chacun à tout moment (voie publique), les lieux publics par destination (bureau, salle d'audience, salle de cours et tribunaux, bars) ouvert au public à certain moment déterminé et aussi les lieux publics par accident, privé en principe mais devenant occasionnellement public par le fait de la présence d'un certain nombre des personnes». Les personnes protégées sont les particuliers.

Il s'agit ici, selon les articles 74 et 75 du code pénal, des personnes. La diffamation doit être dirigée directement ou indirectement contre une personne.

La diffamation existe, chaque fois qu'il existe un fait précis, de nature à causer préjudice à la victime. Ainsi donc, trois éléments sont exigés pour son existence, à savoir: un acte d'imputation ; un fait précis et un préjudice.

D'après SHONGO (2017:67), « imputer un fait à une personne, c'est affirmer que cette personne est l'auteur. En d'autres termes, c'est mettre un fait au compte ou à la charge d'une personne ».

Un acte d'imputation suppose un fait nettement déterminé, c'est-à-dire aussi un fait qui peut faire l'objet d'une preuve. En effet un fait est précis lorsque sa véracité ou sa fausseté peut faire l'objet d'une preuve directe ou indirecte.

A ce qui concerne le préjudice, la loi exige que le fait précis puisse porter atteinte à l'honneur ou à la considération d'une personne ou susceptible de l'exposer au mépris public. C'est-à-dire tout fait dirigé contre la dignité, la loyauté, l'honnêteté, l'estime ou la morale; et aussi toute imputation pouvant compromettre les égards résultant de la position sociale acquise par la victime.

L'élément moral de cette infraction se traduit par le terme employé par la loi: celui qui a méchamment...L'auteur doit être animé par la volonté de nuire ou d'offenser la victime. La peine applicable est de huit jours à un an et une amende de vingt mille francs ou l'une de ces peines seulement.

Comme nous l'avions si bien relevé précédemment les réseaux sociaux sont de plus a plus utilisés à ce jour, ils permettent de créer des forums adapté aux besoins de chacun. On a la possibilité d'avoir diffèrent type de forum à savoir: forum d'échanges avec les proches les collèges, promotion, les étudiants, les élevés, les parents etc.

C'est sur ces forums ou se partage des écrits, des vidéos et photo de tout genre, allant des photos des évènements, des accidents, des faits rigolos. Il peut arriver qu'une personne s'estime victime d'un écrit, une image, d'une vidéo balancée sur les réseaux. En ce sens deux questions peuvent être soulevées à savoir:

1. si on tombe dans le cas d'imputation dommageable ou diffamation?

2. Etant donné qu'un forum a plus de deux personnes peut-t-on parle de la publicité pour prétendre être victime de diffamation?

La lecture que nous donne la définition de LIKULIA BOLONGO quand ce dernier dit que: « aussi les lieux publics par accident, privé en principe mais devenant occasionnellement public par le fait de la présence d'un certain nombre des personnes », nous laisse croire que les réseaux sociaux peuvent aussi être considérés comme un lieu public puisque qu'ayant plusieurs membres.

\section{La responsabilite des medias en cas de delit de presse}

Le délit est civil mais dans le langage courant, le délit est souvent synonyme d'infraction en droit français par exemple est considéré comme délit, toute infraction que les lois punissent de peine correctionnelle ». Mais en droit congolais, les juristes soutiennent que le concept délit ne relève qu'en matière civile d'après les prescrits de l'art 258 Code Civil Congolais Livre III qui dispose que «tout fait quelconque de l'homme qui cause à autrui un dommage, oblige celui par la faute duquel il est arrivé à le réparer ».

Ici, la loi congolaise ne prévoit ni une liste, ni un contenu précis, il est tel du seul moment qu'il cause préjudice à autrui. Mais en République Démocratique du Congo lors qu'on parle de « délit de presse on doit 
vite recourir à l'art 74 de la loi 96-002 du 22 juin 1996 fixant les modalités de l'exercice de la liberté de presse, pour en tirer la définition. L'article cité définit le délit de presse comme «toute infraction écrite ou audiovisuelle ».

Il peut aussi être définit comme un délit d'opinion par les moyen de la presse. Chaque fois que la presse sert de moyen d'expression à une infraction, cette infraction devient un délit de presse.

Quant aux faux bruits, cette infraction est mentionnée à l'art 199 du code pénal Congolais est souvent associée à celle d'imputation dommageable, offense au chef de l'Etat ou outrage à l'autorité. Elle est aussi souvent évoquée lorsque le journaliste est poursuivi pour l'atteinte à la sureté de l'Etat ou divulgation des secrets de défense nationale.

S'agissant de la publicité comme la constituante de diffamation sans pour autant passé par de détail, les propos doivent avoir été publiés c'est-àdire porté à la connaissance d'autrui.

S'agissant de l'allégation ou l'imputation, ici, elles sont appréciées de manière souple par le juge. Selon la définition du terme «allégation», il s'agit d'une assertion, d'une proportion avancée, proposition et mal fondée voire mensongère. L'imputation quant à elle est le fait d'attribuer à quelqu'un une action, un fait, un comportement. Ces deux définitions sont donc très proches, quoique l'allégation soit souvent perçue comme nécessairement du bibitive et l'imputation comme nécessairement péjorative.

En définitive, nous disons que les journalistes aggravent leur responsabilité pénale en cas de violation d'une disposition pénale. Ainsi voyons sa responsabilité civile.

Il sied de noter que la liberté de presse n'est pas une valeur absolue. Si le droit garanti a tous citoyens la libre circulation des moyens de communication, il en fixe aussi les limites. Ainsi pour des raisons de précision, l'existence de certaines limites n'est pas contraire entant que telle, à l'affirmation juridique de la liberté d'expression.

La liberté en ce sens ne pas une licence. Il n'est guère de système juridique qui ne permettent, expressément, de limiter l'exercice des libertés affirmées. Mais ces limitations outre leurs caractères explicites est déterminé, ne peuvent à leurs tour dépassé certaines limites, faute de quoi l'espace de la liberté se réduit comme une peau de chagrin.

Ces limites ou ces bornes méritent d'être considéré sous un double aspect celui de leur motif ou de leur justification.
Dans le même angle d'idée, il se pose la question sur la responsabilité des médias. Faut-il pour autant dédouaner les journalistes et leurs médias lorsqu'ils commettent des erreurs ou des fautes plus ou moins graves? Assurément non. Quand bien même on est un défenseur intrépide de la liberté de la presse. On doit reconnaitre la part de la responsabilité des journalistes dans l'exercice de leurs fonctions. Les sanctions civiles qui sont prises à leurs encontre doivent se référencer aux dispositions du code civil qui traitent du délit.

D'où la référence à l'article 258 CCC L III du décret du 30 Juillet 1888 portant code des contrats, des obligations conventionnelles. Ainsi en s'appuyant sur les codes civiles congolais les journalistes sont responsables civilement pour les faits qu'ils ont commis en agissant comme sociétaire d'une entreprise médiatique ou comme personne physique. La loi souligne que chacun est responsable de dommage qu'il a causé non seulement par ce fait mais encore par sa négligence ou son imprudence (Article 259 CCC L III).

\section{CONCLUSION}

Le journaliste avant d'être un professionnel de la communication médiatique est d'abord une personne humaine.

La constitution consacre certains droits qui le concernent d'une manière particulière, notamment le droit à liberté de pensée, droit à la liberté d'expression, droit à l'information, liberté d'information et d'émission par la radio et la télévision, droit à la protection etc.

La même constitution contient plusieurs dispositions qui rappellent les devoirs du journaliste. Son article 31 recommande au journaliste de veiller sur «le droit de toute personne au respect de sa vie privée et au secret de la correspondance, de la télécommunication et de toute autre forme de communication ». La constitution insiste également sur le respect de la loi, de l'ordre public, des droits d'autrui et des bonnes mœurs.

Les dispositions de la loi $\mathrm{n}^{\circ}$ 96-002 du 29 juin 1996 énumèrent quelques droits fondamentaux reconnus aux journalistes aux articles 8-10, section $3 \mathrm{du}$ $1^{\mathrm{er}}$ chapitre. Nous pouvons citer entre autre: droit à la liberté d'opinion et d'expression, droit à la liberté, à l'indépendance d'esprit, la liberté de choix du titre d'un écrit ou d'une émission.

Certes, il est vrai que la présomption d'innocence s'efface au fur et à mesure que les indices de culpabilité pèsent sur la personne. La détention serait une mesure pouvant empêcher l'auteur présumé de l'infraction de se soustraire de la poursuite aussi longtemps qu'il existe contre lui des indices sérieux de culpabilité. La poursuite pénale d'un journaliste peut se 
Menga Rene Yaosuwa \& Imani Gaya., Sch Bull, Jan 2020; 6(1): 32-40

justifier aux prescrits de la constitution affirmant que « la personne humaine est sacrée, l'Etat a l'obligation de la respecter et de la protéger.

Ainsi, le journaliste auteur présumé de l'infraction perd le bénéfice de la présomption d'innocence lorsque les faits mis à sa charge portent atteinte à l'honneur ou à la considération de la personne. La critique reste libre et nécessaire dans toutes sociétés se prétendant démocratique, seul élément à prendre en compte est les faits de porter atteinte à l'honneur et à la considération de la personne.

Par ricochet, lorsque l'honneur et la considération d'une personne sont dénaturées par voie médiatique, l'Etat est appelé à intervenir en vertu de la prérogative constitutionnelle qui lui est reconnue par le biais de l'action publique nonobstant le principe de la présomption d'innocence. Les délits de presse portent des stigmates psychologiques, professionnels, sociétaux et familiaux. Les acteurs des medias sont dans l'obligation de moyen et de résultat quant à la vérification des contenus des informations qu'ils doivent diffuser.

\section{REFFERENCE}

1. Luzolo Bambi Lessa EJ. Cours de procédure pénale G2 Unikin, 2006-2007.

2. Bille Francis. Medias et société presse Audiovisuel télécommunication, multimédia à télématique, Paris, $7^{\text {ème }}$ éd Mont Chrétien, 1994.

3. Luc, A.T.(2004). Le droit de la presse dans le contexte : étude critique des textes juridiques sur la presse, Kigali, Ed. Universitaire.

4. M. Grawitz Et Pinto. (2008). Méthode en sciences sociales, Paris, Ed. Dalloz, 2008

5. Jeveau. C. (1976). Comprendre la sociologie, Paris, Marabout, 1976

6. Nyabirungu, M. S.(1998). Traité de droit pénal général, Kinshasa, $2^{\mathrm{ème}}$ éd D.E.S, 1998

7. Dr. Emmanuel. (2002). Droit de l'information responsabilité pénale des medias, Paris.

8. Loi $\mathrm{N}^{\circ}$ 04/017 du 30 juillet. (2004). Portant Organisation et Fonctionnement de la Haute Autorité des Medias. 